\title{
Lecciones desde África: Impacto socioeconómico del enclave turístico en Livingstone, Zambia
}

\section{Lessons from Africa: Social-economic impacts of enclave tourism in Livingstone, Zambia}

Percy Mabvuto Ngwira es Primer Secretario de Turismo y Oficial de Enlace de Zambia para la Organización Mundial del Turismo OMT (París, Francia) (tngwira@gmail.com) (https://orcid.org/0000-0001-9616-5245)

\begin{abstract}
Resumen
El presente artículo emplea el paradigma de dependencia para ilustrar los impactos socioeconómicos del turismo de enclave en países en desarrollo utilizando la ciudad de Livingstone en Zambia (África) como caso de estudio. Utilizando fuentes primarias y secundarias, este estudio revela que los inversionistas extranjeros y los turistas internacionales dominan principalmente la industria del turismo en Livingstone. El dominio del sector por parte de inversionistas extranjeros ha resultado en el desarrollo de un paradigma de dependencia de la actividad turística para contribuir significativamente al desarrollo socioeconómico de la ciudad de Livingstone. A pesar de ser acreditado como un sector importante que contribuye al desarrollo socioeconómico y al alivio de la pobreza, el enfoque del paradigma de dependencia del desarrollo turístico en Livingstone ha conducido a vínculos más débiles con la economía doméstica, lo que ha tenido un impacto mínimo en el crecimiento económico general de la ciudad. Además, el turismo en Livingstone se ha vuelto poco competitivo desde una perspectiva comercial debido al dominio de la propiedad extranjera de las principales empresas turísticas que han creado carteles y oligopolios en el negocio, creando así barreras para que las empresas locales compitan efectivamente. Debido a su naturaleza, el sector turístico en Livingstone no se puede declarar como sostenible. Para abordar los problemas del turismo de enclave y promover un desarrollo turístico más inclusivo y beneficioso en Livingstone y en otros destinos turísticos de países en desarrollo, es necesario adoptar políticas y estrategias que garanticen la plena participación de las comunidades locales y un retorno doméstico de las significativas ganancias del turismo. Las estrategias también deben garantizar que el desarrollo turístico tenga fuertes vínculos con el resto de actividades socioeconómicas locales.
\end{abstract}

\begin{abstract}
This paper employs dependency paradigm to illustrate the social-economic impacts of enclave tourism on developing countries using Livingstone town in Zambia, Africa as a case study. Utilising both primary and secondary data sources, the study finds that, foreign investors and international tourists primarily dominate the tourism industry in Livingstone. The domination of the sector by foreign investors has resulted in dependency paradigm tourism development and failure by tourism to significantly contribute to social-economic development Livingstone town. Despite being credited as a significant sector that contributes to the social-economic development and poverty alleviation, dependency paradigm approach to tourism development in Livingstone has led to weaker linkages with domestic economy resulting in minimal impact on the overall economic growth of the town. Additionally, tourism in Livingstone has been rendered uncompetitive from a business perspective due to dominance by foreign ownership of key tourism enterprises who have created cartels in the business, thereby creating barriers for local businesses to compete effectively. Because of its nature, the tourism sector in Livingstone cannot be pronounced as sustainable. To, address problems of enclave tourism and promote more inclusive and beneficial tourism development in Livingstone and other tourism destinations in developing countries, there is need to adopt policies and strategies that ensure full involvement of local communities and the return of significant earnings from tourism. The strategies must also ensure that tourism development has strong linkages with the rest of the local social-economic activities.
\end{abstract}

\section{Palabras clave I keywords}

Turismo de enclave, desarrollo de turismo sostenible, países en desarrollo, Livingstone, Zambia. Enclave tourism, sustainable tourism development, developing countries, Livingstone, Zambia.

Forma sugerida de citar: Mabvuto Ngwira, Percy (2018). Lecciones desde África: Impacto socioeconómico del enclave turístico en Livingstone, Zambia. Retos Revista de Ciencias de la Administración y Economía, 15(8), 35-50.

https://doi.org/10.17163/ret.n15.2018.03 


\section{Introducción}

El presente artículo se basa en el paradigma de dependencia para ilustrar los impactos socioeconómicos del turismo de enclave en países en desarrollo utilizando la ciudad de Livingstone en Zambia (África) como caso de estudio (figura 1).

Podría decirse que el turismo se ha convertido en una de las industrias de mayor crecimiento del siglo XXI en el mundo. En su libro Turismo y política pública, publicado en 1995, John Tribe y Colin Michael Hall reconocen y predicen el continuo crecimiento de la industria del turismo al afirmar que "el turismo es la industria más grande del mundo y se espera que continúe creciendo y manteniendo ese estado en el siglo XXI" (Tribe \& Hall, 1995, p. 1). Dos décadas después de que Hall \& Tribe hicieran esta predicción, el mundo ha sido testigo del crecimiento de esta industria, posiblemente a niveles sin precedentes.

Figura 1. Mapa de Zambia que muestra la ubicación de la ciudad de Livingstone

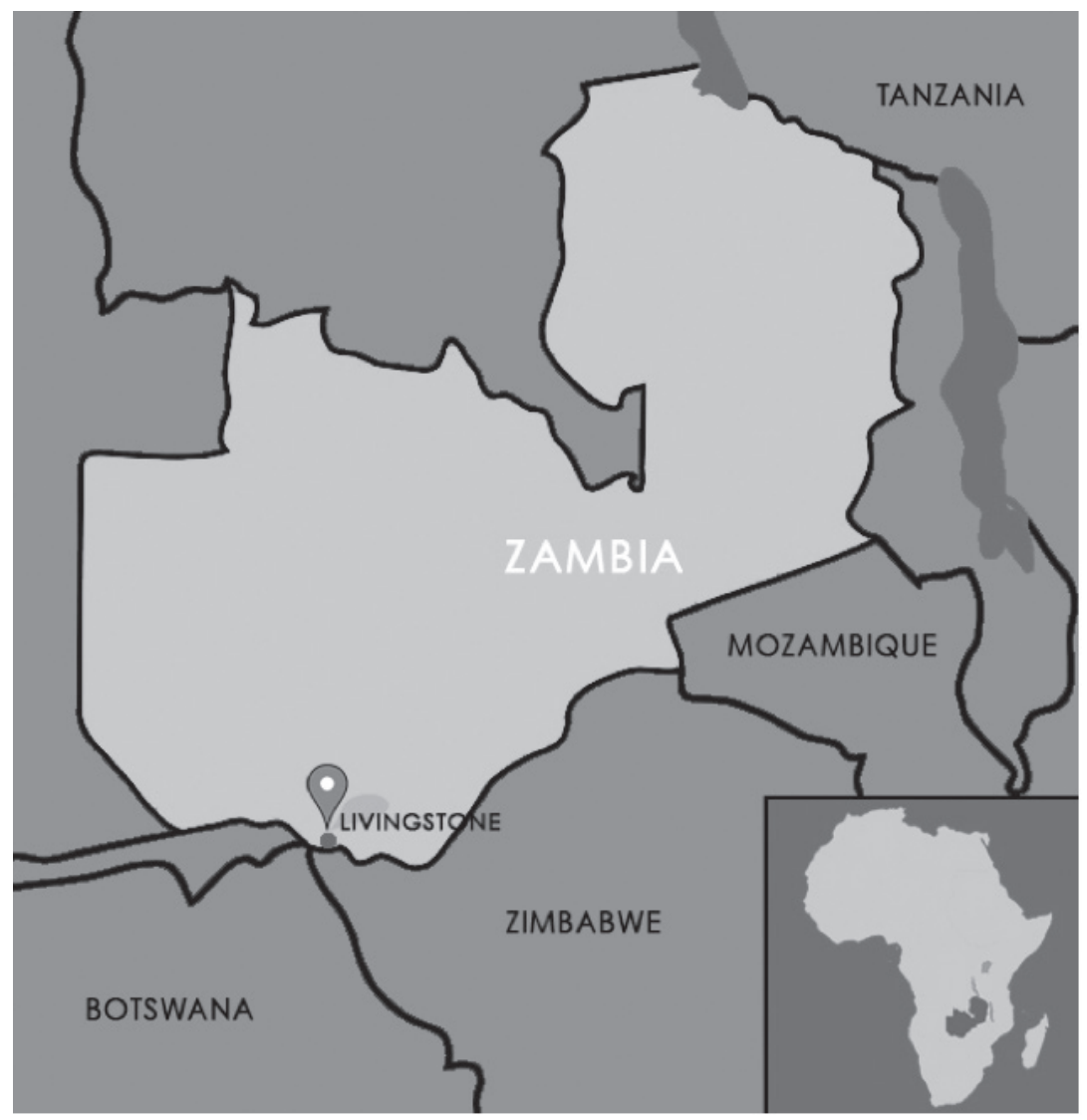

Fuente: map-states.bid 
Según el Consejo Mundial del Viaje y el Turismo (WTTC, 2017), la industria de viajes y turismo se ha convertido en un sector crítico para el desarrollo económico y la creación de empleo en todo el mundo. Solo en 2016, la contribución directa de esta industria a la economía mundial y la creación de empleo representaron USD 2,3 billones y 109 millones de empleos a escala mundial. El sector contribuyó con USD 7,6 billones a la economía del mundo y mantuvo 292 millones de empleos en 2016, teniendo en cuenta sus impactos indirectos e inducidos más amplios. La contribución fue equivalente al 10,2\% del PIB mundial y representa aproximadamente 1 de cada 10 empleos (WTTC, 2017).

En consecuencia, la Organización Mundial del Turismo (OMT) señala que el turismo internacional y los viajes han crecido espectacularmente desde la década de 1970. Esto a pesar de las guerras, la agitación política, los desastres naturales, los sustos médicos, los ataques terroristas, y las crisis económicas y energéticas en diversas partes del mundo (OMT, 2013a).

Además, los informes y registros de la OMT indican que el turismo mundial ha experimentado un crecimiento y una diversificación extendida durante décadas para convertirse en una de las industrias de más rápido crecimiento en el mundo (OMT, 2015). El crecimiento del turismo moderno se ha relacionado diligentemente con el desarrollo e incluye un número cada vez mayor de nuevos destinos. Estas dinámicas han convertido al turismo en un motor clave para el progreso socioeconómico. A pesar de shocks aleatorios, la llegada de turistas internacionales ha mostrado un crecimiento prácticamente constante de 25 millones en 1950 a 278 millones en 1980, 528 millones en 1995 y 1035 millones en 2012 (OMT, 2015).

En cuanto a perspectivas a largo plazo, la OMT ha anticipado que la llegada de turistas internacionales en todo el mundo aumentará anualmente en un 3,3\% de 2010 a 2030, llegando a 1800 millones en 2030. Entre estos años se espera que las visitas a destinos emergentes (+ 4,4\% anual) aumenten al doble del ritmo de las economías avanzadas (+ 2,2\% anual) (puntos destacados de la OMT, 2013b).

Li \& Guo (2017) señalan que, en los países, regiones, pueblos y ciudades en desarrollo, el turismo se considera un catalizador del desarrollo. Por lo tanto, los gobiernos de los países en desarrollo ven a esta actividad como una vía rápida para el crecimiento y el principal contribuyente al desarrollo socioeconómico nacional. Esto se debe a la capacidad del sector para atraer ingresos extranjeros, crear oportunidades de empleo, proporcionar ventajas de balanza de pagos, y contribuir de manera esencial al progreso de infraestructuras que benefician tanto a locales como a visitantes (Akama \& Kieti, 2007).

En Zambia el turismo era casi inexistente cuando el país se independizó de Gran Bretaña en 1964. Investigadores como Teye (1986) afirman que el turismo no fue reconocido como un sector digno de desarrollo en Zambia hasta 1965, después de que este país del sur de África obtuviera su independencia. Arthur (1968) afirma que en 1965 Zambia desarrolló el primer Plan Nacional de Desarrollo. Este plan proyectó un total de 155000 visitantes extranjeros y facturación por un total de USD 6 millones en 1970, basándose en las llegadas de turistas y en los ingresos a principios de los años sesenta. Ngwira \& Musinguzi (2011a) documentan que "desde la independencia hasta 1996, la industria del turismo de Zambia se clasificaba simplemente como sector 
social”. Sin embargo en 1996 el gobierno de Zambia reclasificó el sector turístico, antes considerado social, como un sector económico. Esto se dio al reconocer el potencial del sector para contribuir al desarrollo económico en términos, entre otros, de las ganancias de divisas, el empleo y la generación de ingresos, la contribución a los ingresos del Gobierno, la promoción del desarrollo rural y el papel de catalizador de un desarrollo sostenible. Para el año 2016, podía decirse que la industria del turismo de Zambia había crecido hasta convertirse en el segundo sector económico más grande del país, contribuyendo con un 4\% a su Producto Interno Bruto (Ministerio de Turismo y Artes 2015, 2016).

Gran parte de los turistas internacionales que visitan Zambia van a Livingstone debido a las Cataratas Victoria/Mosi-oa-Tunya, una de las siete maravillas naturales del mundo y Patrimonio de la Humanidad inscrita por la Organización de las Naciones Unidas para la Educación, la Ciencia y la Cultura (UNESCO) en 1989, siendo éstas una atracción turística compartida entre Zambia y Zimbabue. Los factores de atracción turística alternativa de Livingstone son la variedad de prácticas de deportes extremos que ofrece el destino y el rico patrimonio cultural e histórico, complementado con la vida silvestre en el hábitat del Parque Nacional Musi-a-tunya.

La Agencia de Turismo de Zambia, el ala gubernamental de comercialización de la marca país, sugiere que en la última década Livingstone ha recibido más de un cuarto de millón de turistas, casi el doble que la década anterior. En promedio, casi la mitad del total de turistas de Zambia visita Livingstone. El crecimiento del turismo en Livingstone ha provocado el desarrollo de una diversidad de infraestructuras e instalaciones asociadas, como hoteles, albergues, campamentos de safaris y casas de huéspedes. El único aeropuerto en Livingstone fue mejorado y la pista ampliada para permitir el aterrizaje de aviones más grandes.

Aunque la ciudad de Livingstone ha recibido parte justa de su desarrollo de turístico y la infraestructura en la última década y ha atraído a muchos inversionistas extranjeros, podría decirse que todo el negocio en Livingstone está en gran parte en manos de los extranjeros. Investigadores como Mbaiwa (2002) se refieren a desarrollos tales que conducen a una economía turística en auge basada en lo que se percibe internacionalmente como un destino «nuevo» y «exótico». Ahn, Lee \& Shafer (2002) afirman que las instalaciones y los programas de turismo se desarrollan para crear cambios, como el aumento del ingreso personal o de ingresos tributarios a una región anfitriona. Sin embargo el turismo, como cualquier desarrollo, también puede producir cambios que eliminen oportunidades o amenacen la calidad de vida, como es el caso de estudio.

Investigaciones previas sobre el desarrollo del turismo en Zambia, en general, y en Livingstone, en particular (Teye, 1988; Husbands, 1989; Rakner, 2003; y Muuka et al., 2006), se centraron en los impactos económicos positivos del turismo, tales como ingresos en divisas, la creación de empleos y el desarrollo de infraestructura, sin necesariamente extender su investigación a cuestiones de alivio de la pobreza y a las implicaciones socioeconómicas del turismo en el destino, o investigando la distribución equitativa de los beneficios del lado de las partes interesadas. El actual crecimiento y desarrollo del turismo en Livingstone, por lo tanto, plantea cuestiones de sostenibilidad en relación a los beneficios socioeconómicos. 
Wall (1997) explica que el desarrollo sostenible permite que el turismo sea económicamente viable, culturalmente apropiado y ecológicamente sensible. El enfoque del paradigma de dependencia del desarrollo socioeconómico en Livingstone presenta un interesante tema de debate entre economistas y expertos en turismo. Ngwira \& Musinguzi (2011b) afirman que, a pesar de atraer a tantos inversionistas extranjeros en el sector del turismo, Livingstone sigue siendo una de las muchas ciudades con altos niveles de pobreza en Zambia.

El paradigma de dependencia es uno de los marcos que se ha utilizado para describir el turismo en los países en desarrollo (Meyer, 2013). El concepto de paradigma de dependencia se basa en la creencia de que los recursos fluyen de una "periferia» de Estados pobres y subdesarrollados a un «núcleo» de Estados ricos, enriqueciéndolos a expensas de los primeros. En el contexto del turismo, el marco se basa en el entendimiento de que el turismo en los países en desarrollo depende principalmente de la demanda de los países desarrollados y se organiza a partir de ellos. Este escenario crea un tipo de turismo conocido como «turismo de enclave» (Mbaiwa 2003, Mbaiwa, 2005a). En consecuencia, el propósito de este artículo es utilizar el paradigma de dependencia para explicar el impacto socioeconómico del desarrollo del turismo de enclave en los países en desarrollo utilizando el destino Livingstone en Zambia como ejemplo. El artículo también utiliza el concepto de desarrollo sostenible para evaluar la sostenibilidad del turismo en Livingstone.

\section{Desarrollo del turismo sostenible}

El concepto de desarrollo sostenible resulta de una cosmovisión que considera que la supervivencia, el progreso y el mantenimiento continuo de la comunidad humana dependen de la salud y viabilidad continua de los sistemas de soporte vital de la Tierra (Keiner, 2005). El desarrollo sostenible implica procesos de cambio fundamental en nuestras instituciones sociales y acciones individuales. La motivación de este cambio se relaciona con enfrentar los desafíos propios del siglo XXI, tales como una conciencia global de que la tierra es finita, y de que todos los sistemas de soporte vital del planeta, incluyendo los sistemas sociales y económicos a nivel mundial, están interconectados y son interdependientes.

La publicación del informe Brundtland «Nuestro Futuro Común» de 1987, ampliamente criticado en algunos círculos por carecer de detalles específicos, marcó un punto de inflexión en el pensamiento sobre el medio ambiente, el desarrollo y la gobernanza. La Comisión Mundial sobre Medio Ambiente y Desarrollo patrocinada por las Naciones Unidas (WCED), dirigida por Gro Harlem Brundtland, emitió un llamado audaz para recalibrar los mecanismos institucionales a escala mundial, nacional y local para promover un desarrollo económico que garantice la seguridad, el bienestar y la supervivencia misma del planeta (WCED, 1987, p. 23). Sneddon et al. (2006) enfatizan que el llamado al desarrollo sostenible fue una redirección del proyecto de la ilustración, una respuesta pragmática a los problemas de nuestros tiempos.

El informe Brundtland (1987, s/p) también proporcionó la definición ampliamente aceptada de desarrollo sostenible, indicando que "el desarrollo sostenible es un desarrollo que satisface las necesidades del presente sin comprometer la capacidad 
de las generaciones futuras para satisfacer sus propias necesidades". La Organización Mundial del Turismo (OMT, 1998) documenta que la Conferencia de las Naciones Unidas sobre Medio Ambiente y Desarrollo, popularmente conocida como la «Cumbre de la Tierra» celebrada en 1992 en Brasil, estimuló aún más la necesidad de un desarrollo turístico sostenible, lo que quedó expuesto como una línea fundamental en la «Agenda 21 , adoptada por la conferencia.

Después de la conferencia de Río, las organizaciones y gobiernos de muchas naciones comenzaron a adoptar la sostenibilidad como una política de desarrollo fundamental. La OMT fue una de las primeras organizaciones internacionales que adoptó el enfoque sostenible del desarrollo del turismo, predicando principios sostenibles en todas sus directrices de desarrollo y planificación del turismo. Hunt (1992, p. 2) sugirió que el concepto de desarrollo del turismo sostenible es "una de las ideas más saludables del turismo". Sin embargo, el concepto no se entiende fácilmente y, como política, ciertamente no se implementa cómodamente (Haider \& Johnston, 1992; Burr \& Walsh, 1994). Butler (1993) sugirió que una definición viable de desarrollo sostenible en el contexto del turismo podría entenderse como un turismo que sigue siendo viable durante un período indefinido y no degrada ni altera el entorno (humano y físico) en el que existe, hasta el punto de prohibir el desarrollo exitoso y el bienestar de otras actividades y procesos. De acuerdo con las sugerencias de Butler -referidas ut supra-, la OMT ha definido el «turismo sostenible» como el "desarrollo turístico que satisface las necesidades de los turistas actuales y las regiones anfitrionas a la vez que protege y mejora las oportunidades para el futuro". Está previsto que conduzca a la gestión de todos los recursos de forma tal que se puedan satisfacer las necesidades económicas, sociales y estéticas, manteniendo a la vez la integridad cultural, los procesos ecológicos esenciales, la diversidad biológica y los sistemas de soporte vital (Organización Mundial del Turismo 1998).

\section{Metodología}

La presente investigación utiliza un diseño de investigación cualitativa, basándose en los datos recopilados mediante entrevistas realizadas por el autor entre enero de 2017 y julio de 2017. Si bien la investigación se basó en información obtenida de fuentes primarias y secundarias, el estudio se enfocó y se basó principalmente en datos secundarios. La recopilación de datos secundarios se orientó en el uso de la información obtenida a través de las literaturas recopiladas de documentos de políticas gubernamentales, informes, y todo tipo de documento publicado y no publicado sobre turismo en Livingstone, en particular, y Zambia, en general. La recopilación de datos primarios implicó la administración de entrevistas estructuradas y semiestructuradas con un complemento de cuestionarios para 35 interesados del sector turístico de Livingstone. Se administraron entrevistas estructuradas y semiestructuradas tanto a inversionistas extranjeros y locales de turismo, residentes locales, y a empleados domésticos que trabajan en diversas empresas de turismo.

Adicionalmente, la recopilación de datos primarios se complementó con discusiones formales mantenidas con funcionarios del gobierno central y local en Livingstone. Estos funcionarios fueron seleccionados aleatoriamente de instituciones 
tales como la Oficina Regional del Ministerio de Turismo y Arte, el Ayuntamiento de Livingstone, la Oficina del Distrito de Trabajo del Ministerio de Trabajo, la Oficina del Comisionado de Distrito, la Oficina de la Agencia de Turismo de Zambia en Livingstone, y la Asociación de Turismo de Livingstone, una asociación para operadores turísticos con sede en Livingstone.

Las entrevistas con operadores turísticos y residentes locales se centraron en cuestiones de propiedad de instalaciones turísticas, oportunidades de empleo, beneficios salariales, y los impactos del turismo en su vida social. Por otro lado, las entrevistas formales y los cuestionarios administrados a los funcionarios del gobierno central y local de Livingstone se centraron en cuestiones de políticas, leyes laborales, leyes de inversión, distribución equitativa de los beneficios derivados del turismo, y la participación de la comunidad local en la planificación y el desarrollo del turismo en Livingstone.

\section{Resultados}

Los impactos socioeconómicos del turismo de enclave en Livingstone no son únicos con respecto a otros destinos turísticos en muchos países en desarrollo alrededor del mundo (Shaw y Shaw; 1999, Mbaiwa, 2005b, Anderson, 2011; Naidoo \& Sharpley; 2016). La ciudad de Livingstone, antiguamente la capital de Zambia, pasó de ser un pequeño asentamiento en Old Drift en 1905 (Arrington, 2010) a ser una moderna ciudad africana con una población de 139, 509 habitantes y una densidad de población de 201 personas por kilómetro cuadrado (CSO, 2010). En cuanto al desarrollo socioeconómico, puede afirmarse que Livingstone ha experimentado una transformación vacilante de una ciudad industrial próspera combinada con una industria turística en auge, para convertirse en una de las ciudades más económicamente desfavorecidas del país con altas tasas de desempleo.

Históricamente, en el pasado Livingstone fue una próspera ciudad en Zambia, todas las personas ricas y famosas vivían en Livingstone y, aparte de ser la capital de Zambia y el principal destino turístico, en su momento fue una ciudad industrial con una de las mejores fábricas de ensamblaje de automotores en el país (Rogerson, 2005). Sin embargo, la ciudad comenzó a perder su poder económico después de que la capital se trasladó a Lusaka, situación que se agravó con el colapso económico nacional en la década de 1980, que pareció haber puesto el último clavo en lo que alguna vez fue una ciudad económicamente próspera en Zambia.

Desde la recesión económica de la década de 1980, Livingstone ha luchado por recuperarse y, en general, la economía de la ciudad, al igual que la economía nacional, ha dependido mucho del sector informal para su desarrollo económico. Shah (2012) observa que "uno de los principales desafíos económicos de Zambia es un sector informal en constante crecimiento". Gerxhani (2004) señala que este sector típicamente se caracteriza por su facilidad de ingreso (cualquiera puede iniciar un negocio cuando lo desee) bajos niveles de habilidades, tecnología intensiva en mano de obra, y pequeñas empresas. Sin embargo, su contribución de valor real a la economía es muy baja.

El sector informal en Zambia emplea al 90\% de la fuerza de trabajo y ha proliferado en los últimos años, alcanzando aproximadamente a más de un millón de 
empresas informales, que en su mayoría son de base rural; involucran actividades agrícolas realizadas por agricultores con poca capacitación y que generan bajos ingresos (Shah, 2012). Como Shah observó correctamente, las pequeñas empresas y la economía informal son las principales actividades económicas de Livingstone. Gran parte de la economía informal en Livingstone implica: comercio transfronterizo, extracción de piedra, venta de curiosidades y artesanías, pequeñas tiendas, vendedores callejeros o máquinas expendedoras. Mientras tanto, otros residentes de Livingstone se dedican a la agricultura a pequeña escala, o producen y venden diversos productos agrícolas, desde hortalizas hasta crianza de ganado, pollos y cabras.

En consecuencia, la Cámara de Comercio de Livingstone afirma que este alto porcentaje de negocios opera fuera del alcance de los impuestos del gobierno/consejo y de los sistemas de recuperación de costos. Por lo tanto, no contribuye a los sistemas de generación de ingresos del gobierno.

En términos de desarrollo turístico, se puede sostener que Livingstone ha sido testigo de un crecimiento constante en el desarrollo del turismo en las últimas dos décadas. Sin embargo, este nivel de desarrollo se ha mantenido a un paso de tortuga en comparación con otras ciudades turísticas en la misma región, como Sudáfrica, Botswana e incluso la ciudad fronteriza de Victoria Falls sobre el río Zambezi en Zimbabwe. Como lo defienden McLachlan \& Binns, (2014) "Livingstone, la capital del turismo de Zambia, ha experimentado un rápido crecimiento tanto económico como social, convirtiéndose en un punto turístico dentro del bloque sur de África”. Según los registros de la Agencia de Registro de Patentes y Empresas (PACRA), una agencia ejecutiva semiautónoma del Ministerio de Comercio, Intercambio e Industria de Zambia, más de 157 empresas de turismo se habían registrado solo en Livingstone durante el primer trimestre de 2014.

El Gobierno afirma que el importante desarrollo turístico en Livingstone en las últimas dos décadas es indiscutible (Ministerio de Turismo y Artes, Departamento de Turismo, 2016). La ciudad recibió un apoyo masivo del gobierno en el desarrollo de infraestructura hacia el período previo a la copresidencia de la Asamblea General de la OMT de 2013. Los fondos estuvieron a disposición para mejoras en las carreteras del municipio, y para la expansión de la pista en el aeropuerto internacional de Livingstone, capaz de aterrizar aviones tan grandes como un Boeing 777 y un Airbus A380. Además, el aeropuerto recibió fondos para una nueva terminal internacional (Lusaka Times, 2011). Se mejoró el mercado del Distrito Comercial Central de Livingstone y se construyó una nueva terminal de autobuses. Este rango sin precedentes de desarrollo de infraestructura dentro de la ciudad ha hecho que el turismo se convierta en un foco no solo para Livingstone, sino a nivel nacional para el desarrollo económico y social (Ministerio de Turismo y Artes, Departamento de Turismo, 2015).

Livingstone también ha sido testigo de un desarrollo sin paralelo en el área de alojamiento de turistas, a la que muchos jugadores, tanto locales como extranjeros, han 
subido a bordo para establecer instalaciones de alojamiento de calidad en la ciudad. Podría decirse que la llegada del Sun International Resort Hotel significó el inicio del desarrollo del turismo moderno en Livingstone.

El gobierno de Zambia percibe este desarrollo en Livingstone como una oportunidad para que la gente local se involucre directamente en las empresas turísticas, dando al sector el potencial para convertirse en una industria que sea social y económicamente sostenible.

Es probable que el turismo en Zambia se desarrolle completamente centrado en Livingstone, y desde hace bastante tiempo Livingstone sigue siendo un destino de visita obligada en el país para los turistas locales e internacionales. La Tabla (1) a continuación muestra las llegadas de turistas internacionales por origen a la ciudad de Livingstone para el período entre 2012 y 2016. El turismo en Livingstone y Zambia en general depende principalmente de turistas extranjeros. El turismo interno en el país todavía está en sus primeras etapas.

Tabla 1. Llegadas de turistas a Livingstone por origen 2012-2016

\begin{tabular}{|l|r|r|r|r|r|}
\hline \multirow{2}{*}{\multicolumn{1}{c|}{ Origen }} & \multicolumn{5}{c|}{ Año } \\
\cline { 2 - 6 } & $\mathbf{2 0 1 2}$ & $\mathbf{2 0 1 3}$ & \multicolumn{1}{c|}{$\mathbf{2 0 1 4}$} & \multicolumn{1}{c|}{$\mathbf{2 0 1 5}$} & \multicolumn{1}{c|}{$\mathbf{2 0 1 6}$} \\
\hline Sudáfrica & 54701 & 60192 & 79120 & 66021 & 88933 \\
\hline África del Este & 40644 & 42937 & 45735 & 48533 & 51331 \\
\hline África del Norte & 568 & 606 & 652 & 598 & 844 \\
\hline África Occidental & 1028 & 1508 & 1608 & 705 & 1608 \\
\hline África Central & 34251 & 45428 & 51271 & 58913 & 65500 \\
\hline Europa & 65087 & 70595 & 69757 & 55919 & 134081 \\
\hline América & 20682 & 26638 & 29250 & 21413 & 43801 \\
\hline Asia y Oceanía & 27956 & 30097 & 32710 & 35323 & 57936 \\
\hline Total & 244917 & 278001 & 310103 & 287425 & 444034 \\
\hline
\end{tabular}

Fuente: Ministerio de Turismo y Artes (2016)

En términos de facturación, Livingstone también se considera el principal contribuyente a los ingresos obtenidos del sector turístico. Entre 2012 y 2016, los ingresos totales anuales directos de la ciudad de Livingstone obtenidos de varias empresas de turismo oscilaron entre 148841075 y 188000 000, respectivamente. La Tabla (2) muestra un aumento del período de cinco años en la generación de ingresos de los subsectores del turismo para los años 2012 a 2016. 
Tabla 2. Recibos del turismo de Livingstone 2012-2016

\begin{tabular}{|l|r|r|r|r|r|}
\hline \multirow{2}{*}{ Subsector } & \multicolumn{6}{|c|}{ Año y cantidades en USD } \\
\cline { 2 - 7 } & 2012 USD & 2013 USD & 2014 USD & 2015 USD & 2016 USD \\
\hline Alojamiento & 561321,00 & 60571624 & 62150653 & 66638473 & 70900017 \\
\hline Viaje & 44174611 & 47668409 & 48911068 & 52442874 & 55796606 \\
\hline Tours & 25953158 & 28005810 & 28735888 & 30810870 & 32781231 \\
\hline $\begin{array}{l}\text { Alquiler de } \\
\text { automóviles }\end{array}$ & 14726759 & 15891508 & 16305781 & 17483201 & 18601255 \\
\hline Otros & 7854445 & 8475658 & 8696608 & 9324579 & 9920888 \\
\hline Total & 148841075 & 160613011 & 164800000 & 176700000 & 188000000 \\
\hline
\end{tabular}

Fuente: Ministerio de Turismo y Artes (2016)

En relación con el empleo, la ciudad de Livingstone tiene la mayor fuerza de trabajo empleada en el sector del turismo en Zambia. Uno de cada tres empleados que viven en Livingstone trabaja para empresas turísticas o negocios relacionados con el turismo. La Figura (2) a continuación muestra un período de cinco años desde 2012 hasta 2016 del número de empleos o trabajos creados por el sector turístico en Livingstone.

Figura 2. Estado de empleo turístico en Livingstone 2012-2016

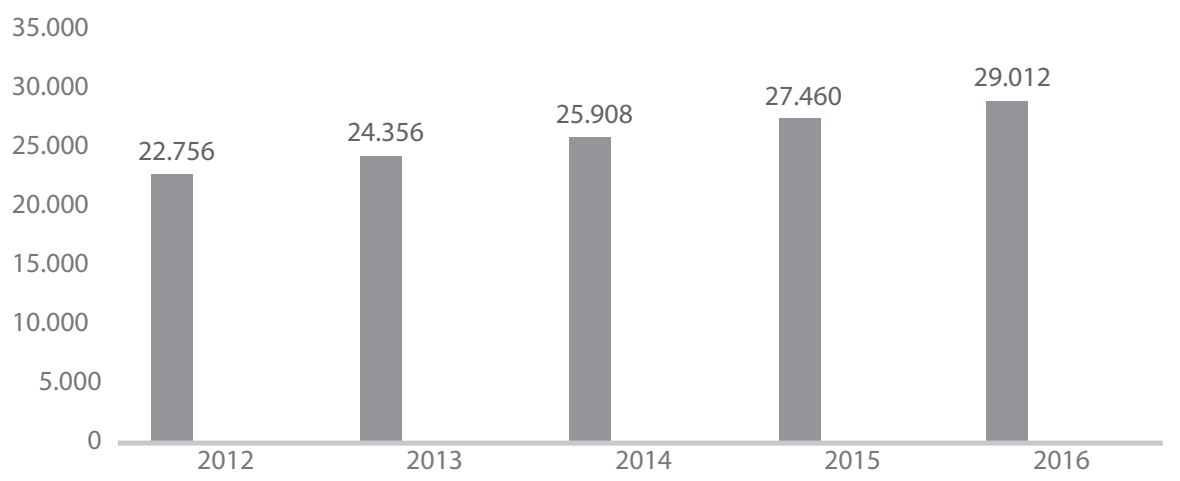

Fuente: Ministerio de Turismo y Artes (2016)

El pilar del turismo en Livingstone es debatiblemente el Musi-ao-tunya / Cataratas Victoria, un producto turístico y patrimonio mundial compartido con Zimbabwe. Situadas a unos $10 \mathrm{~km}$ de la ciudad de Livingstone, las Cataratas Victoria son uno de los productos turísticos más visitados en Zambia; es una de las atracciones 
turísticas obligadas de África. La Tabla (3) a continuación muestra los números de visitantes turísticos locales y extranjeros al sitio del Patrimonio Mundial de las Cataratas Victoria para el período de 2005 a 2010.

Tabla 3. Visitantes locales y extranjeros

a las Cataratas Victoria 2005-2010

\begin{tabular}{|l|c|c|c|}
\hline \multicolumn{1}{|c|}{ Año } & Visitantes locales & $\begin{array}{c}\text { Visitantes } \\
\text { extranjeros }\end{array}$ & Total \\
\hline 2010 & 79162 & 41038 & 120200 \\
\hline 2011 & 68734 & 40690 & 109424 \\
\hline 2012 & 82224 & 44267 & 126491 \\
\hline 2013 & 178580 & 68234 & 246814 \\
\hline 2014 & 110790 & 71980 & 182770 \\
\hline 2015 & 158778 & 88986 & 147764 \\
\hline Total & 678268 & 355195 & 1033463 \\
\hline
\end{tabular}

Fuente: NHCC (2016)

Aunque los turistas locales superan el número de turistas internacionales que visitan las Cataratas Victoria, en términos de ingresos, se generan más ingresos de turistas extranjeros ya que muchos turistas locales a las Cataratas Victoria son estudiantes de escuela que van de manera gratuita y que, sin embargo, son incluidos en las estadísticas.

Una evaluación de la contribución del turismo al desarrollo socioeconómico requiere una investigación sobre los vínculos hacia atrás y hacia adelante entre el turismo y otros sectores, una comprensión de la ubicación espacial de las actividades turísticas, y la identificación de los benefactores de sus impactos económicos y de otro tipo (Briedenhann y Eugenia 2004). Por lo tanto, la principal preocupación de cualquier destino turístico en los países en desarrollo es su capacidad de tener fuertes vínculos con los sectores sociales y económicos nacionales como agricultura, manufactura, construcción, comercio mayorista y minorista, instalaciones de alojamiento y restaurantes, transporte, banca y servicios de seguros, agua y electricidad, y servicios sociales y personales.

\section{Discusiones}

Los resultados de este estudio, tal como lo ilustran los datos primarios de las entrevistas de campo realizadas por el autor en Livingstone y respaldados por datos secundarios, indican que debido a la naturaleza de la industria turística de Zambia (Livingstone) (control externo y gestión de las empresas turísticas, leyes laborales y de inversión del gobierno más débiles, participación limitada de la comunidad local, altas tasas de fuga y vínculos débiles con la economía nacional), el sector del turismo no ha jugado un papel útil para contribuir al desarrollo socioeconómico local. 
Además, los datos recopilados y analizados revelan que la contribución del turismo de Zambia al Producto Interno Bruto (PIB) sigue siendo mínima, principalmente debido a los vínculos hacia adelante y atrás más débiles y a una fuerte dependencia de fuentes externas. Por ejemplo, el Ministerio de Turismo y Artes, Departamento de Turismo (2016) produjo un informe que demuestra el impacto del turismo en la economía de Zambia. El informe indica que el turismo contribuyó con el 3,4\% del PIB en 2015. Mientras tanto, el Séptimo Plan Nacional de Desarrollo solo ha capturado algunos sectores seleccionados de toda la industria del turismo y registra el 1,4\% (Hoteles y Restaurantes) como una contribución al Producto Interno Bruto Nacional. A pesar de los datos poco confiables sobre la contribución real del turismo al PIB de Zambia, entrevistas con funcionarios del Ministerio de Turismo confirman que la industria del turismo de Zambia no está funcionando y contribuyendo a los ingresos del gobierno como se esperaba, citando sus vínculos débiles con el resto de la economía doméstica, junto con algunas regulaciones débiles.

Con respecto a quién recibe el mayor beneficio de las empresas de inversión turística y el turismo en Livingstone, se descubrió que la comunidad se sentía excluida, ya que acusaban a los inversionistas extranjeros y al Estado de ser los principales beneficiarios de los ingresos de la industria del turismo. Deseaban poder beneficiarse más a nivel del hogar.

Con respecto a la inversión, los resultados indican que Zambia tenía políticas de inversión muy ambiciosas. Sin embargo, tenía algunas deficiencias; por ejemplo, la Ley de la Agencia de Desarrollo de Zambia estipula que todos los inversionistas extranjeros deben traer al menos a cinco altos directivos para que trabajen en sus negocios, un tipo de política que no favorece al profesional local. Las políticas de inversión también carecían de una dirección clara con respecto a la contribución de los inversionistas extranjeros de turismo al desarrollo de la economía local en las áreas donde invierten; esto se ha dejado a discreción de los inversionistas. Además, se descubrió que a los inversionistas extranjeros se les habían dado demasiados incentivos que habían llevado a conceptos erróneos entre la población local.

Adicionalmente, los datos disponibles de la autoridad de registro de la compañía PACRA muestran que en Livingstone el $80 \%$ de las empresas turísticas son de propiedad extranjera, los ciudadanos poseen el $18 \%$, y el $2 \%$ es propiedad conjunta de ciudadanos y no ciudadanos. Esto sugiere que las empresas y los inversionistas extranjeros tienen una influencia de aproximadamente el $80 \%$ del negocio del turismo en Livingstone. Rogerson (2011) realizó estudios anteriores y similares que confirman que las empresas y los inversionistas extranjeros dominan principalmente la industria del turismo en Livingstone y Zambia. Esta situación tiene un impacto social y económico negativo en el destino de acogida. Este estado de la cuestión no está en línea con los principios del desarrollo sostenible que enfatizan la igualdad de acceso y la participación de la población local en el uso de los recursos turísticos. Por lo tanto, una industria del turismo sostenible debe ser sensible a las necesidades y aspiraciones de la población de acogida y brindarles la oportunidad de participar en emprendimientos comerciales (Liu, 2003).

Con respecto a las leyes laborales, los resultados indican que en Zambia las leyes laborales son de alguna manera débiles, especialmente cuando se trata de proteger a los profesionales locales. Este estudio encontró que una función que los inversionistas 
del turismo extranjero pueden desempeñar en Livingstone es contribuir al alivio de la pobreza ofreciendo oportunidades de trabajo a la población local. Sin embargo, los inversionistas en turismo extranjero fueron criticados por ofrecer empleos de bajo salario, como jardineros, mucamas, camareros, conductores, recepcionistas, porteros y cocineros, a la mayoría de los empleados locales, mientras que los trabajos de administración se otorgan a extranjeros. Se argumentó que estos empleos otorgados a la población local no tienen un alto valor monetario que contribuiría al alivio de la pobreza y al desarrollo económico sostenible en Livingstone. Por ejemplo, se descubrió que el salario mínimo de Zambia para los trabajadores de la industria del turismo estaba muy por debajo del nivel de vida promedio de la población local.

Además de las contribuciones del turismo al Producto Interno Bruto (PIB) Nacional y de ofrecer oportunidades de empleo a la población local, el otro factor de valor relacionado con el impacto socioeconómico del turismo en los países en desarrollo es la propiedad de las empresas turísticas. Este estudio encontró que en Livingstone la mayoría de las empresas turísticas son propiedad de inversionistas extranjeros, una situación que parece haber perpetuado el «turismo de enclave» o el «turismo capturado». Es turismo capturado en el sentido de que estas compañías de propiedad extranjera han creado carteles comerciales (Connor \& Lande, 2012) que han dado como resultado que los pocos inversionistas locales no participen plenamente o compitan de manera eficiente con sus colegas inversionistas extranjeros.

Además, este estudio encontró que debido al tipo de desarrollo turístico en Livingstone, el sector tuvo un impacto negativo en la generación de ingresos, ya que gran parte de los ingresos generados por el turismo en Livingstone se repatriaron fuera del país, y muchas empresas de turismo extranjeras operan a través de paquetes turísticos que permiten a sus clientes pagar sus servicios en el país de origen, reduciendo así el efecto de derrame económico.

El estudio también encontró que la dominación de turistas o visitantes extranjeros en Livingstone ha provocado negatividad en el comportamiento social de la población local, especialmente los jóvenes. Los datos principales de las entrevistas indican que muchas personas locales en Livingstone estaban involucradas en conductas sociales negativas, como abuso de alcohol y participación en actividades sexuales que han resultado en un aumento de enfermedades de transmisión sexual como el VIH/SIDA.

\section{Conclusión}

Este artículo ha analizado cuestiones relacionadas con el turismo sostenible como una herramienta para el desarrollo socioeconómico local en los países en desarrollo, utilizando el estudio de caso de Livingstone, una ciudad en Zambia. Esto fue demostrado por datos primarios de entrevistas de campo realizadas en Livingstone, y respaldado por datos secundarios. La presente investigación encontró que la naturaleza de la industria del turismo en Livingstone se caracteriza por el control externo y la gestión de las empresas turísticas, leyes laborales y de inversión gubernamentales débiles, participación limitada de la comunidad local, altas tasas de fuga y vínculos débiles con la economía. El presente estudio concluye que la industria del turismo no ha desempeñado un papel efectivo en el desarrollo socioeconómico local en Livingstone. 
Aunque existen impactos socioeconómicos más bien negativos con respecto al turismo de enclave en Livingstone, este también tiene algunas ventajas, aunque son bastante difíciles de validar. El crecimiento del turismo en las últimas dos décadas en Livingstone ha estimulado el desarrollo de una variada infraestructura e instalaciones aliadas. Esto incluye la construcción de centros comerciales, reparación y modernización de las calles e instalaciones de comunicación del municipio, expansión de la pista en el aeropuerto internacional de Livingstone, la construcción de una nueva terminal internacional, la modernización del mercado del distrito comercial central de Livingstone, y la construcción de una nueva terminal de autobuses. Livingstone también ha sido testigo del desarrollo de más instalaciones de alojamiento de diferentes gamas. A través de sus vínculos hacia atrás, las empresas mayoristas y minoristas también se han establecido para ofrecer diversos bienes y servicios a la industria del turismo. El turismo en Zambia se ha convertido en el tercer sector más importante del país después de la minería y la agricultura. A pesar de los impactos socioeconómicos positivos del turismo de enclave en Livingstone, la industria no ha podido promover un desarrollo económico sostenible y la reducción de la pobreza en la mayor parte de la ciudad.

Dado que el turismo en Livingstone depende principalmente de inversionistas extranjeros y turistas, el turismo interno y la participación ciudadana en el turismo son muy bajos. La industria también se caracteriza por la repatriación de los ingresos del exterior del país y vínculos débiles con la economía nacional. Debido a que los impactos negativos superan a los positivos, el turismo en Livingstone podría describirse como "turismo capturado" o turismo de enclave, que en gran parte está controlado por negocios y turistas extranjeros.

Para abordar los problemas del turismo de enclave y promover un desarrollo turístico más inclusivo y beneficioso en Livingstone y otros países en desarrollo, es necesario adoptar políticas y estrategias que garanticen la plena participación de las comunidades locales y el retorno de las ganancias sustanciales del turismo. Las estrategias también deben garantizar que el desarrollo turístico tenga fuertes vínculos con el resto de actividades económicas.

\section{Bibliografía}

Ahn, B., Lee B. \& Shafer, C.S. (2002). Operationalizing sustainability in regional tourism planning: an application of the limits of acceptable change framework. Tourism Management, 23(1), 1-15. https://doi.org/10.1016/S0261-5177(01)00059-0

Akama, J. S. \& Kieti, D. (2007). Tourism and socio-economic development in developing countries: A case study of Mombasa Resort in Kenya. Journal of sustainable tourism, 15(6), 735-748. https://doi.org/10.2167/jost543.0

Anderson, W. (2011). Enclave tourism and its socio-economic impact in emerging destinations. Anatolia, 22(3), 361-377. https://doi.org/10.1080/13032917.2011.633041

Arrington, A. L. (2010). Competing for tourists at Victoria Falls: A historical consideration of the effects of government involvement. Development Southern Africa, 27(5), 773-787. https:// doi.org/10.1080/0376835X.2010.522838

Arthur, L. D. (1968). Zambia's Tourism: The Way Ahead. A Tourism Development Study for Zambia. London: Arthur D. Little, Inc 
Burr, S.W. \& Walsh J. A. (1994). A hidden value of sustainable tourism development. Trends in Sustainable Rural Tourism Development, 31(1), 9-13.

Butler, R. W. (1993). Tourism-an evolutionary perspective. In J.G. Nelson, R.W. Butler, and G. Wall (Eds.) Tourism and Sustainable Development Monitoring, Planning, Managing (pp.27-43). Waterloo, Ontario: Department of Geography, University of Waterloo.

Central Statistical Office (CSO) (2010). Zambia 2010 Census of Population and Housing. Lusaka, Zambia.

Connor, J.M. \& Lande R. H. (2012). Cartels as rational business strategy: crime pays. Cardozo Law Review, 34. http://dx.doi.org/10.2139/ssrn.1917657

Gerxhani, K. (2004). The Informal Sector in Developed and Less Developed Countries: a literature survey. Public Choice, 120(3-4), 267-300. https://goo.gl/4p2bU9

Haider, W. \& Johnston, M. (1992). Tourism and community development. Annals of Tourism Research, 19(3), 580-583. https://doi.org/10.1016/0160-7383(92)90146-G

Hunt, J.D. (1992). Rural tourism: new focus on a traditional industry. Western Wildlands, 18(3), 2-3.

Husbands, W. (1989). Social status and perception of tourism in Zambia. Annals of tourism research, 16(2), 237-253. https://doi.org/10.1016/0160-7383(89)90070-4

Keiner, M. (2005). Re-emphasizing sustainable development-The concept of 'Evolutionability'. Environment, Development and Sustainability, 6(4), 379-392. https://doi.org/10.1007/ s10668-005-5737-4

Li, R. \& Guo, L. (2017). The effect of tourism development on poverty alleviation: a literature review. Tourism Tribune, 32(6), 28-37.

Liu, Z. (2003). Sustainable tourism development: A critique. Journal of Sustainable Tourism, 11(6), 459-475. https://doi.org/10.1080/09669580308667216

Lusaka Times (2011). Livingstone International Airport to expand. https://goo.gl/TxvdcV (242017)

Mbaiwa, J. E. (2002). The socio-economic and environmental impacts of tourism development in the Okavango Delta, Botswana: A baseline study. Harry Oppenheimer Okavango Research Centre, University of Botswana, Maun.

Mbaiwa, J. E. (2003). The socio-economic and environmental impacts of tourism development on the Okavango Delta, north-western Botswana. Journal of Arid Environments, 54(2), 447-467. https://doi.org/10.1006/jare.2002.1101

Mbaiwa, J. E. (2005a). Enclave tourism and its socio-economic impacts in the Okavango Delta, Botswana. Tourism Management, 26(2), 157-172. https://doi.org/10.1016/j.tourman.2003.11.005

Mbaiwa, J. E. (2005b). The problems and prospects of sustainable tourism development in the Okavango Delta, Botswana. Journal of Sustainable Tourism, 13(3), 203-227. https://doi. org $/ 10.1080 / 01434630508668554$

McLachlan, S. \& Binns, T. (2014). Tourism, development and corporate social responsibility in Livingstone, Zambia. Local Economy, 29(1-2), 98-112. https://doi. org/10.1177/0269094214520624

Meyer, D. (2013). Exploring the duality of structure and agency-the changing dependency paradigms of tourism development on the Swahili coast of Kenya and Zanzibar. Current Issues in Tourism, 16(7-8), 773-791. https://doi.org/10.1080/13683500.2013.785487

Ministry of Tourism and Arts (2015). Strategic Plan 2014-2016. Lusaka, Zambia: Government Printers.

Ministry of Tourism and Arts (2016). 2015 Tourism Statistical Digest. Lusaka: Zambia: Kwacha House.

Muuka, G. N., Hendon, G., Niffenegger, P. \& Muuka, L.W. (2006). When tourism has the potential to turn a nation around: the case of Zambia in the 21st century. Consortium Journal of Hospitality \& Tourism, 10(2). 
Naidoo, P. \& Sharpley, R. (2016). Local perceptions of the relative contributions of enclave tourism and agritourism to community well-being: The case of Mauritius. Journal of Destination Marketing \& Management, 5(1), 16-25. https://doi.org/10.1016/j.jdmm.2015.11.002

Ngwira, P. \& Musinguzi, D. (2011). Tourism and poverty alleviation in Zambia: opportunities, challenges and the way forward. The Joint Symposium on "Sustainability and Quality-of-Life in Tourism: Tasks for Consumers, Industry, Policy and Academia", May 2011.

Rakner, L. (2003). Political and economic liberalisation in Zambia 1991-2001. Uppsala: Nordic Africa Institute.

Rogerson, C. M. (2011) Tourism Food Supply Linkages in Zambia: Evidence from the African Safari Lodge Sector. Tourism Review International, 15(1-2), 21-35. https://doi.org/10.372 7/154427211X13139345020174

Rogerson, C. M. (2005). The emergence of tourism-led local development: The example of Livingstone, Zambia. Africa Insight, 35(4), 112-120. http://hdl.handle.net/10520/EJC17446

Shah, M. (2012). The Informal Sector in Zambia: Can it Disappear? Should it Disappear? London: International Growth Centre.

Shaw, B. J. \& Shaw, G. (1999). 'Sun, Sand and Sales': Enclave Tourism and Local Entrepreneurship in Indonesia. Current Issues in Tourism, 2(1), 68-81. https://doi. org/10.1080/13683509908667844

Sneddon, C., Howarth, R.B. \& Norgaard, R.B. (2006). Sustainable development in a post-Brundtland world. Ecological Economics, 57(2), 253-268. https://doi.org/10.1016/j.ecolecon.2005.04.013

Teye, V. B. (1986). Liberation wars and tourism development in Africa: The case of Zambia. Annals of Tourism Research, 13(4), 589-608. https://doi.org/10.1016/0160-7383(86)90004-6

Teye, V.B. (1988). Geographic factors affecting tourism in Zambia. Annals of Tourism Research, 15(4), 487-503. https://doi.org/10.1016/0160-7383(88)90045-X

Tribe, J. \& Colin M.H. (1995). Tourism and public policy. London: Cengage Learning EMEA.

United Nations World Tourism Organisation (1998). Sustainable Development of Tourism, Definition. https://goo.gl/Rf30Nv (10-11- 2017).

United Nations World Tourism Organisation (2013). Tourism Highlights. https://goo.gl/LTZSL7 (12-11-2017).

United Nations World Tourism Organisation (2015). Tourism - an economic and social phenomenon. https://goo.gl/5Qm1vo (11-11-2017).

Wall, G. (1997). Is ecotourism sustainable? Environmental management, 21(4), 483-491. https:// doi.org/10.1007/s002679900044

WCED, UNCED (1987). Our common future. World Commission on Environment and Development Oxford University Press.

WTTC (2017). Travel and Tourism Economic Impact Report 2017. London, United Kingdom. 